\title{
Radial boundary values of Poisson integrals on infinite-dimensional balls
}

\section{Oleh Lopushansky*}

\section{${ }^{\text {"Correspondence: }}$}

ovlopusz@ur.edu.pl

Faculty of Mathematics and Natural

Sciences, Rzeszów University,

1 Pigonia str., Rzeszów, 35-310,

Poland

\section{Springer}

\begin{abstract}
We consider a Gelfand triple $E^{\prime} \rightarrow H \rightarrow E$, so that $E$ is a separable complex Banach space with dual $E^{\prime}$, and $H$ is its dense Hilbert subspace. We investigate the problem of analytic extensions on an open ball $\mathcal{Q} \subset E^{\prime}$ and their radial boundary values in the Hardy spaces $\mathcal{H}_{\mu}^{p}(1 \leq p \leq \infty)$ using the Poisson integrals on the unitary group $U(\infty)$ over $\mathrm{H}$ endowed with an invariant probability measure $\mu$. For this purpose, we construct a Poisson-type kernel with the help of the symmetric Fock space $\Gamma$ generated by $H$ and prove that the set of radial boundary values of these analytic functions entirely coincides with $\mathcal{H}_{\mu}^{p}$.
\end{abstract}

MSC: Primary 46T12; 46G20; secondary 46E50

Keywords: Poisson integrals on infinite-dimensional balls; radial boundary values; Wiener measures on groups; Hardy spaces on infinite-dimensional groups

\section{Introduction}

A goal of the current work is to describe a certain type of complex-valued Poisson kernels generated by symmetric Fock spaces and associated Poisson integrals in the case of Hardy spaces in infinite-dimensional settings. This allows us to get a solution of the radial boundary problem for the corresponding analytic extensions.

The main results of the paper are as follows. We consider a Gelfand triple $E^{\prime} \rightarrow H \rightarrow E$ consisting of a separable complex Banach space $E$ with dual $E^{\prime}$ and a densely embedded Hilbert subspace $H$. In Section 2 we investigate the space $\mathcal{H}^{2}$ of analytic functions on an open ball $\mathcal{Q}$ in $E^{\prime}$, which is conjugate-linearly isometric to the symmetric Fock space $\Gamma$ generated by $H$. Its orthogonal polynomial basis is described in Section 3.

In Section 4 we introduce an invariant probability Wiener-type measure $\mu$ on the infinite-dimensional unitary group $U(\infty)=\bigcup U(j)$, irreducibly acting in $H$, where $U(j)$ are subgroups of unitary $(j \times j)$-matrices. This measure is defined as the projective limit of probability Haar measures $\mu_{j}$ on $U(j)$ and is a group analog of probability Wiener measures on Banach spaces, which were introduced by Gross [1]. Its description substantially uses the theory of invariant measures over infinite-dimensional unitary groups developed by Neretin [2] and Olshanski [3].

Using the known Prokhorov criterion and the Schwartz theorem, we show in Theorem 4.1 that $\mu$ is invariant under the right actions of $U^{2}(\infty)$ over $U(\infty)$ and that $\mu$ is a weak limit of a subsequence $\left(\mu_{j_{k}}\right)$. In Theorem 4.3 a concentration property of the sequence $\left(\mu_{j}\right)$ is established.

(c) 2016 Lopushansky. This article is distributed under the terms of the Creative Commons Attribution 4.0 International License (http://creativecommons.org/licenses/by/4.0/), which permits unrestricted use, distribution, and reproduction in any medium, provided you give appropriate credit to the original author(s) and the source, provide a link to the Creative Commons license, and indicate if changes were made. 
The Hardy spaces $\mathcal{H}_{\mu}^{p}(1 \leq p \leq \infty)$ of $L_{\mu}^{p}$-integrable complex-valued functions are described in Section 5. An orthogonal polynomial basis in the Hilbert space $\mathcal{H}_{\mu}^{2}$ is given by Theorem 5.1. Integral formulas for analytic extensions to the open ball $\mathcal{Q} \subset E^{\prime}$ by means of a group generalization of the Paley-Wiener map associated with $\mu$ are established in Theorems 6.2 and 8.1 .

The tools are applied in Section 8 to describe the radial boundary values of functions defined by the integral Poisson formula. In the space $\mathcal{H}_{\mu}^{p}$ with $1 \leq p<\infty$ this problem is described by Theorem 8.3. The existence of weak radial boundary values in $\mathcal{H}_{\mu}^{\infty}$ is established in Theorem 8.4.

Note that the Hardy spaces $\mathcal{H}_{\mu}^{p}$ of analytic functions on infinite-dimensional polydiscs were considered in the works of Cole and Gamelin [4] and Ørted and Neeb [5]. Similar spaces on more general infinite-dimensional domains that are not necessarily polydiscs were investigated by Pinasco and Zalduendo [6], Carando et al. [7], and others.

\section{On analyticity associated with Gelfand triples}

Let $(E,\|\cdot\|)$ be a complex separable Banach space, and $E^{\prime}$ be its normed dual. Consider a complex separable Hilbert space $H$ with scalar product $\langle\cdot \mid \cdot\rangle$ and norm $\|\cdot\|_{H}=\langle\cdot \mid \cdot\rangle^{1 / 2}$ such that the sequence of linear mappings $E^{\prime} \rightarrow H \stackrel{J}{\rightarrow} E$ forms a Gelfand triple with a continuous dense embedding $J$.

Denote $B:=\left\{h \in H:\|h\|_{H}<1\right\}$ and $S:=\left\{h \in H:\|h\|_{H}=1\right\}$. The Hermitian dual $H^{*}$ of $H$ is identified with $H$ via the conjugate-linear isomorphism ${ }^{*}: H^{*} \rightarrow H^{* *}=H$ such that $\eta(h)=\left\langle h \mid \eta^{*}\right\rangle$ for all $h \in H, \eta \in H^{*}$.

Since the embedding $J$ is dense and $H$ is reflexive, the transpose mapping $J^{t}: E^{\prime} \rightarrow H^{*}$ is injective continuous and has the dense range $\mathscr{R}\left(J^{t}\right)$.

Fix an orthonormal basis $\left(e_{j}\right)_{j \in \mathbb{N}}$ in $H$ so that every functional $e_{j}^{*}=\left\langle\cdot \mid e_{j}\right\rangle$ belongs to $\mathscr{R}\left(J^{t}\right)$. Following [6], we define the involution ${ }^{\dagger}: h \mapsto h^{\dagger}:=\sum \bar{e}_{j}^{*}(h) e_{j}$ for any $h=\sum e_{j}^{*}(h) e_{j} \in H$. If $\eta \in H^{*}$, then $\eta^{\dagger}$ is defined so that $\left(\eta^{\dagger}\right)^{*}=\left(\eta^{*}\right)^{\dagger}$, that is, $\eta\left(h^{\dagger}\right)=\bar{\eta}^{\dagger}(h)$. These involutions in $H$ and $H^{*}$ are isometric and depend on the basis chosen.

Thus, we have the Gelfand triple $E^{\prime} \stackrel{J^{*}}{\rightarrow} H \stackrel{J}{\rightarrow} E$ with an injective covariance operator $J \circ J^{*} \in \mathscr{L}\left(E^{\prime}, E\right)$ such that $J^{*}:={ }^{*} \circ^{\dagger} \circ J^{t}$, where the injective mapping $J^{*}$ is continuous and has the dense range $\mathscr{R}\left(J^{*}\right)$. The unbounded inverse $A=\left(J \circ J^{*}\right)^{-1}$ is defined on the dense domain $\mathscr{D}(A)=H$ in $E$. Denote by

$$
\mathcal{Q}:=\left\{z \in E^{\prime}: h=J^{*} z \in B\right\}
$$

the inverse image of the open unit ball $B$ with respect to the injective mapping $J^{*}: E^{\prime} \rightarrow H$. Clearly, the set $\mathcal{Q}$ is the open unit ball in the dual space $E^{\prime}$ endowed with the norm $\|z\|_{J^{*}}:=$ $\left\|J^{*} z\right\|_{H}$ induced from $H$.

It is important to note that the set $\mathcal{Q}$ is also open with respect to the norm topology in $E^{\prime}$ because this topology is stronger than that induced by $J^{*}$, so it contains all open sets induced from $H$.

Let $H^{\otimes n}$ be the complete $n$th tensor power of $H$ endowed with the scalar product $\left\langle\psi_{n} \mid \psi_{n}^{\prime}\right\rangle=\left\langle h_{1} \mid h_{1}^{\prime}\right\rangle \cdots\left\langle h_{n} \mid h_{n}^{\prime}\right\rangle$ for all $\psi_{n}=h_{1} \otimes \cdots \otimes h_{n}, \psi_{n}^{\prime}=h_{1}^{\prime} \otimes \cdots \otimes h_{n}^{\prime} \in H^{\otimes n}$ and $h_{i}, h_{i}^{\prime} \in H(i=1, \ldots, n)$.

As $\sigma:\{1, \ldots, n\} \mapsto\{\sigma(1), \ldots, \sigma(n)\}$ runs through all $n$-element permutations, the complete symmetric $n$th tensor power $H^{\odot n}$ is defined as the range of $H^{\otimes n}$ under the orthogonal projector $S_{n}: \psi_{n} \mapsto h_{1} \odot \cdots \odot h_{n}:=(n !)^{-1} \sum_{\sigma} h_{\sigma(1)} \otimes \cdots \otimes h_{\sigma(n)}$. 
As usual, the symmetric Fock space is defined to be the orthogonal sum

$$
\Gamma=\bigoplus_{n \in \mathbb{Z}_{+}} H^{\odot n}, \quad H^{\odot 0}=\mathbb{C}
$$

of all series $\psi=\bigoplus_{n} \psi_{n}$ convergent with respect to the norm $\|\cdot\|_{\Gamma}=\langle\cdot \mid \cdot\rangle^{1 / 2}$ defined by the scalar product $\left\langle\psi \mid \psi^{\prime}\right\rangle=\sum\left\langle\psi_{n} \mid \psi_{n}^{\prime}\right\rangle$.

The set of elements $h^{\otimes n}:=h \otimes \cdots \otimes h=h \odot \cdots \odot h:=h^{\odot n}$ with any $h \in H$ is total in $H^{\odot n}$ by virtue of the polarization formula for symmetric tensor products $h_{1} \odot \cdots \odot h_{n}=$ $\left(2^{n} n !\right)^{-1} \sum_{\theta_{1}, \ldots, \theta_{n}= \pm 1} \theta_{1} \cdots \theta_{n} h^{\otimes n}$ with $h=\sum_{k=1}^{n} \theta_{k} h_{k}$ for any $h_{1}, \ldots, h_{n} \in H$ (see, e.g., [8], Section 1.5).

Let us consider the $\Gamma$-valued function with a total range

$$
\mathcal{Q} \ni z \mapsto\left(1-J^{*} z\right)^{-\otimes 1}:=\sum_{n \in \mathbb{Z}_{+}} h^{\otimes n}, \quad h=J^{*} z \in B, \quad h^{\otimes 0}=1,
$$

which is analytic because $\left\|(1-h)^{-\otimes 1}\right\|_{\Gamma}^{2}=\sum\|h\|_{H}^{2 n}=\left(1-\|h\|_{H}^{2}\right)^{-1}<\infty$. Using this function, we define the Hilbert space of analytic complex-valued functions in the variable $z \in \mathcal{Q}$, associated with the symmetric Fock space $\Gamma$, as

$$
\mathcal{H}^{2}:=\left\{\psi^{\star}(z)=\left\langle\left(1-J^{*} z\right)^{-\otimes 1} \mid \psi\right\rangle: \psi \in \Gamma\right\}, \quad\left\langle\psi^{\star} \mid \varphi^{\star}\right\rangle_{\mathcal{H}^{2}}:=\langle\varphi \mid \psi\rangle
$$

The space $\mathcal{H}^{2}$ is endowed with the Hilbertian norm $\left\|\psi^{\star}\right\|_{\mathcal{H}^{2}}:=\|\psi\|_{\Gamma}$. Note that $\psi^{\star}(z)=$ $\left(\psi^{\star} \circ A\right)(h)$ for all $h=J^{*} z \in B$. The mapping $\psi \mapsto \psi^{\star}$ is a conjugate-linear isometry from $\Gamma$ on $\mathcal{H}^{2}$.

Functions $\psi^{\star} \in \mathcal{H}^{2}$ are analytic in the variable $z \in \mathcal{Q}$, as a composition of the analytic $\Gamma$-valued function $z \mapsto\left(1-J^{*} z\right)^{-\otimes 1}$ and the linear continuous functional $\psi^{*}=\langle\cdot \mid \psi\rangle$ (see, e.g., [9], Proposition 2.4.2).

\section{Orthogonal homogenous polynomials}

Denote by $\lambda=\left(\lambda_{1}, \ldots, \lambda_{j}\right) \in \mathbb{N}^{j}$ with $\lambda_{1} \geq \lambda_{2} \geq \cdots \geq \lambda_{j}>0$ a partition of $n \in \mathbb{N}$, that is, $n=$ $|\lambda|:=\lambda_{1}+\cdots+\lambda_{j}$. Any $\lambda$ may be identified with a Young diagram of length $\ell(\lambda)=j$. Let $\mathbb{Y}$ denote all Young diagrams, and $\mathbb{Y}_{n}:=\{\lambda \in \mathbb{Y}:|\lambda|=n\}$. Assume that $\mathbb{Y}$ includes the empty partition $\emptyset=(0,0, \ldots)$.

Let $\mathbb{N}_{*}^{\ell(\lambda)}:=\left\{l=\left(l_{1}, \ldots, l_{\ell(\lambda)}\right) \in \mathbb{N}^{\ell(\lambda)}: l_{j} \neq l_{k}, \forall j \neq k\right\}$. An orthogonal basis in $H^{\odot n}$ is formed by the system of symmetric tensor products

$$
e^{\odot \mathbb{Y}_{n}}=\bigcup\left\{e_{l}^{\odot \lambda}:=e_{l_{1}}^{\otimes \lambda_{1}} \odot \cdots \odot e_{l_{\ell(\lambda)}}^{\otimes \lambda_{\ell(\lambda)}}:(\lambda, l) \in \mathbb{Y}_{n} \times \mathbb{N}_{*}^{\ell(\lambda)}\right\}, \quad e_{l}^{\odot \emptyset}=1,
$$

with the norm (see [10], Section 2.2.2)

$$
\left\|e_{l}^{\odot \lambda}\right\|_{\Gamma}=\sqrt{\lambda ! / n !}, \quad \text { where } \lambda !:=\lambda_{1} ! \cdot \ldots \cdot \lambda_{\ell(\lambda)} !
$$

Then $e^{\odot \mathbb{Y}}:=\bigcup\left\{e^{\odot \mathbb{Y}_{n}}: n \in \mathbb{Z}_{+}\right\}$forms an orthogonal basis in $\Gamma$.

Throughout the paper we assume that there exists a unique sequence $\left(z_{j}\right) \subset E^{\prime}$ such that the elements $J^{*} z_{j}=e_{j}$ form an orthonormal basis of $H^{*}$ dual to $\left(e_{j}\right)$. To any index pair 
$(\lambda, \imath) \in \mathbb{Y}_{n} \times \mathbb{N}_{*}^{\ell(\lambda)}$, we uniquely assign the $n$-homogenous polynomial

$$
\zeta_{l}^{\lambda}(z):=\prod_{k=1}^{\ell(\lambda)} \zeta_{l_{k}}^{\lambda_{k}}(z)=\left\langle h^{\otimes n} \mid e_{l}^{\odot \lambda}\right\rangle, \quad h=J^{*} z \in H, \quad \zeta_{l}^{\emptyset} \equiv 1,
$$

considered as a function in the variable $z \in E^{\prime}$ and defined via the Fourier coefficients $\zeta_{j}(z):=\left\langle J^{*} z \mid e_{j}\right\rangle$ of an element $h=J^{*} z \in H$. In other words, $\zeta_{l}^{\lambda}(z)=\left(\zeta_{l}^{\lambda} \circ A\right)(h)$ where $\zeta_{j}(z)=\left\langle h \mid e_{j}\right\rangle$.

Lemma 3.1 The system of $n$-homogeneous polynomials in the variable $z \in E^{\prime}$,

$$
\zeta^{\mathbb{Y}}=\left\{\zeta_{l}^{\lambda}(z)\left\|e_{l}^{\odot \lambda}\right\|_{\Gamma}^{-1}:(\lambda, \iota) \in \mathbb{Y} \times \mathbb{N}_{*}^{\ell(\lambda)}\right\}
$$

with norms $\left\|\zeta_{l}^{\lambda}\right\|_{\mathcal{H}^{2}}=\left\|e_{l}^{\odot \lambda}\right\|_{\Gamma}$ forms an orthonormal basis in $\mathcal{H}^{2}$. Every function $\psi^{\star} \in \mathcal{H}^{2}$ for any $z \in \mathcal{Q}$ has the following Fourier expansion with respect to $\zeta^{\mathbb{Y}}$ :

$$
\psi^{\star}(z)=\sum_{(\lambda, l) \in \mathbb{Y} \times \mathbb{N}_{*}^{\ell(\lambda)}} \tilde{\psi}^{\star}(\lambda, l) \zeta_{l}^{\lambda}(z), \quad \tilde{\psi}^{\star}(\lambda, l):=\left\|e_{l}^{\odot \lambda}\right\|_{\Gamma}^{-2}\left\langle\psi^{\star} \mid \zeta_{l}^{\lambda}\right\rangle_{\mathcal{H}^{2}} .
$$

Proof It suffices to observe that the following orthogonality relation holds:

$$
\left\langle\zeta_{l}^{\lambda} \mid \zeta_{J}^{\mu}\right\rangle_{\mathcal{H}^{2}}=\left\langle e_{J}^{\odot \mu} \mid e_{l}^{\odot \lambda}\right\rangle= \begin{cases}\left\|e_{l}^{\odot \lambda}\right\|_{\Gamma}^{2}: & l=J, \lambda=\mu, \\ 0: & l \neq J \text { or } \lambda \neq \mu .\end{cases}
$$

Taking into account that $J^{*} z=\sum \zeta_{j}(z) e_{j}$ and using the tensor multinomial theorem and (3.1), we obtain the following Fourier decomposition with respect to the basis $e^{\odot \mathbb{Y}}$ in $\Gamma$ :

$$
\begin{aligned}
\left(1-J^{*} z\right)^{-\otimes 1} & =\sum_{n \in \mathbb{Z}_{+}}\left(J^{*} z\right)^{\otimes n} \\
& =\sum_{n \in \mathbb{Z}_{+}}\left(\sum_{k \in \mathbb{N}} \zeta_{k}(z) e_{k}\right)^{\otimes n}=\sum_{(\lambda, l) \in \mathbb{Y} \times \mathbb{N}_{*}^{\ell(\lambda)}} \frac{\zeta_{l}^{\lambda}(z) e_{l}^{\odot \lambda}}{\left\|e_{l}^{\odot \lambda}\right\|_{\Gamma}^{2}}
\end{aligned}
$$

for all $z \in \mathcal{Q}$. Applying this, we conclude that every analytic function $\psi^{\star} \in \mathcal{H}^{2}$ with $\psi=$ $\bigoplus_{n} \psi_{n} \in \Gamma\left(\psi_{n} \in H^{\odot n}\right)$ has the Taylor expansion at zero

$$
\psi^{\star}(z)=\sum_{n \in \mathbb{Z}_{+}}\left\langle\left(J^{*} z\right)^{\otimes n} \mid \psi_{n}\right\rangle, \quad z \in \mathcal{Q},
$$

where

$$
\left\langle\left(J^{*} z\right)^{\otimes n} \mid \psi_{n}\right\rangle=\sum_{(\lambda, l) \in \mathbb{Y}_{n} \times \mathbb{N}_{*}^{\ell(\lambda)}} \frac{\left\langle e_{l}^{\odot \lambda} \mid \psi_{n}\right\rangle}{\left\|e_{l}^{\odot \lambda}\right\|_{\Gamma}^{2}} \zeta_{l}^{\lambda}(z)
$$

are Hilbert-Schmidt polynomials in the variable $h=J^{*} z \in H$ with any $z \in E^{\prime}$.

Lemma 3.2 Each analytic function $\psi^{\star} \in \mathcal{H}^{2}$ can be uniquely written as

$$
\psi^{\star}(z)=\left\langle\psi^{\star}(\cdot)|\mathcal{C}(\cdot, z)|_{\mathcal{H}^{2}}=\left\langle\psi^{\star}(\cdot) \mid \mathcal{P}(\cdot, z)\right\rangle_{\mathcal{H}^{2}}, \quad z, z^{\prime} \in \mathcal{Q}\right.
$$

where $\mathcal{C}\left(z^{\prime}, z\right)=\left\langle\left(1-J^{*} z^{\prime}\right)^{-\otimes 1} \mid\left(1-J^{*} z\right)^{-\otimes 1}\right\rangle$ and $\mathcal{P}\left(z^{\prime}, z\right)=\left|\mathcal{C}\left(z^{\prime}, z\right)\right|^{2} / \mathcal{C}(z, z)$. 
Proof From (3.3) it follows that the complex-valued function $\mathcal{C}\left(z^{\prime}, z\right)$ in the variable $z \in \mathcal{Q}$ with fixed $z^{\prime} \in \mathcal{Q}$ belongs to $\mathcal{H}^{2}$. Using that $J^{*} z=\sum \zeta_{j}(z) e_{j}$, we obtain

$$
\begin{aligned}
\mathcal{C}\left(z^{\prime}, z\right) & =\sum_{n \in \mathbb{Z}_{+}}\left\langle\left(J^{*} z^{\prime}\right)^{\otimes n} \mid\left(J^{*} z\right)^{\otimes n}\right\rangle=\frac{1}{1-\left\langle J^{*} z^{\prime} \mid J^{*} z\right\rangle} \\
& =\sum_{n \in \mathbb{Z}_{+}}\left(\sum_{j \in \mathbb{N}} \zeta_{j}\left(z^{\prime}\right) \bar{\zeta}_{j}(z)\right)^{n}=\sum_{(\lambda, l) \in \mathbb{Y} \times \mathbb{N}_{*}^{\ell(\lambda)}} \frac{\zeta_{l}^{\lambda}\left(z^{\prime}\right) \bar{\zeta}_{l}^{\lambda}(z)}{\left\|e_{l}^{\odot \lambda}\right\|_{\Gamma}^{2}} .
\end{aligned}
$$

Expanding any $\psi^{\star} \in \mathcal{H}^{2}$ in the orthogonal series with respect to $\zeta^{\mathbb{Y}}$, we obtain (3.2). Substituting (3.2) into formula (3.4) and applying Lemma 3.1, we get

$$
\begin{aligned}
\left\langle\psi^{\star}\left(z^{\prime}\right) \mid \mathcal{C}\left(z^{\prime}, z\right)\right\rangle_{\mathcal{H}^{2}} & =\left\langle\sum_{(\lambda, l)} \frac{\zeta_{l}^{\lambda}\left(z^{\prime}\right)\left\langle\psi^{\star} \mid \zeta_{l}^{\lambda}\right\rangle_{\mathcal{H}^{2}}}{\left\|e_{l}^{\odot \lambda}\right\|_{\Gamma}^{2}} \mid \sum_{(\lambda, l)} \frac{\zeta_{l}^{\lambda}\left(z^{\prime}\right) \bar{\zeta}_{l}^{\lambda}(z)}{\left\|e_{l}^{\odot \lambda}\right\|_{\Gamma}^{2}}\right\rangle \\
& =\sum_{(\lambda, l)} \frac{\zeta_{l}^{\lambda}(z)\left\langle\psi^{\star} \mid \zeta_{l}^{\lambda}\right\rangle_{\mathcal{H}^{2}}}{\left\|e_{l}^{\odot \lambda}\right\|_{\Gamma}^{2}} .
\end{aligned}
$$

So, the first equality in (3.4) holds. If $\omega^{\star}\left(z^{\prime}\right):=\left\langle\psi^{\star}(\cdot) \mid \mathcal{C}\left(z^{\prime}, \cdot\right)\left[\mathcal{C}\left(z^{\prime}, z^{\prime}\right)\right]^{-1} \mathcal{C}\left(\cdot, z^{\prime}\right)\right\rangle_{\mathcal{H}^{2}}$, then $\omega^{\star}(z)=\psi^{\star}(z)$ for all $z \in \mathcal{Q}$. As a result, we obtain

$$
\begin{aligned}
\psi^{\star}(z) & =\left\langle\omega^{\star}(\cdot)|\mathcal{C}(\cdot, z)|_{\mathcal{H}^{2}}\right. \\
& =\left\langle\mathcal{C}(z, \cdot)[\mathcal{C}(z, z)]^{-1} \psi^{\star}(z)|\mathcal{C}(\cdot, z)|_{\mathcal{H}^{2}}=\left\langle\psi^{\star}(\cdot)|\mathcal{P}(\cdot, z)|_{\mathcal{H}^{2}} .\right.\right.
\end{aligned}
$$

Hence, the second equality in (3.4) holds. Finally, the totality in $\Gamma$ of elements $\left(1-J^{*} z\right)^{-\otimes 1}$ with any $z \in \mathcal{Q}$ yields the uniqueness of these representations.

\section{Invariant Wiener measures on $U(\infty)$}

We still assume that the orthonormal basis $\left(e_{j}\right)$ of $H$ lies in the range of $J^{*}: E^{\prime} \rightarrow H$, that is, there exist $\left(z_{j}\right) \subset E^{\prime}$ such that $J^{*} z_{j}=e_{j}$.

Let $U(\infty)=\bigcup U(j)$ be the infinite-dimensional unitary matrix group with unit $\mathbb{1}$. The group $U(\infty)$ acts irreducibly on $H$. Denote $U^{2}(\infty):=U(\infty) \times U(\infty)$ and $U^{2}(j):=U(j) \times$ $U(j)$. The right action on $U(\infty)$ (similarly, on $U(j))$ is defined as

$$
u \cdot g=w^{-1} u v \quad \text { for all } u \in U(\infty), g=(v, w) \in U^{2}(\infty)
$$

Following [2, 3], we write every $u_{j} \in U(j)$ with $j>1$ in the block matrix form $u_{j}=\left[\begin{array}{cc}v_{j-1} & a \\ b & t\end{array}\right]$ with $t \in \mathbb{C}$ corresponding to the partition $j=(j-1)+1$ so that $v_{j-1}$ is a $(j-1) \times(j-1)$ matrix. Consider the projective limit $\lim _{\leftarrow} U(j)$ taken with respect to the Livšic-type mapping (which is not a group homomorphism)

$$
\pi_{j-1}^{j}: u_{j}=\left[\begin{array}{cc}
v_{j-1} & a \\
b & t
\end{array}\right] \mapsto u_{j-1}= \begin{cases}v_{j-1}-\left[a(1+t)^{-1} b\right]: & t \neq-1 \\
v_{j-1}: & t=-1\end{cases}
$$

from $U(j)$ on $U(j-1)$, which is Borel and surjective and is commuted with the right action of $U^{2}(j-1)$ (see [2], Proposition 0.1, [3], Lemma 3.1). In particular, it follows that $\pi_{j-1}^{j}:\left[\begin{array}{cc}v_{j-1} & 0 \\ 0 & 1\end{array}\right] \mapsto v_{j-1}$ for all $v_{j-1} \in U(j-1)$. 
Let $\pi_{j}: \lim _{\longleftarrow} U(j) \ni\left(u_{j}\right) \mapsto u_{j} \in U(j)$ be the projection, so that $\pi_{j-1}=\pi_{j-1}^{j} \circ \pi_{j}$.

In what follows, every $U(j)$ is identified with its range under the natural inclusion $U(j) \leftrightarrow U(\infty)$ that assigns to any $u_{j} \in U(j)$ the block matrix $\left[\begin{array}{cc}u_{j} & 0 \\ 0 & \mathbb{1}\end{array}\right] \in U(\infty)$, and let $U(\infty)$ be endowed with the topology of inductive limit under the natural inclusions $U(j-1) \rightarrow U(j)$. Accordingly, $\pi_{j-1}^{j}$ are defined over $U(\infty)$ as block matrices transformations. Let $\pi_{j}^{k}:=$ $\pi_{j}^{j+1} \circ \cdots \circ \pi_{k-1}^{k}$ for $j<k$ and $\pi_{j}^{k}$ for $j=k$ be the identical mapping over $U(\infty)$.

Let us consider the dense injective mapping $\tau: U(\infty) \rightarrow \lim U(j)$ that to any $u_{k} \in U(k)$ assigns the unique stabilized sequence $\left(u_{j}\right)$ such that (see [3], n. 4)

$$
\tau: U(k) \ni u_{k} \mapsto\left(u_{j}\right) \in \lim _{\longleftarrow} U(j), \quad u_{j}= \begin{cases}\pi_{j}^{k}\left(u_{k}\right): & j<k, \\
u_{k}: & j=k, \\
{\left[\begin{array}{cc}
u_{k} & 0 \\
0 & \mathbb{1}
\end{array}\right]:} & j>k .\end{cases}
$$

Denote by $U_{\tau}(\infty)$ the group $U(\infty)$ endowed with the induced topology under the mapping $\tau: U(\infty) \leftrightarrow \lim _{\leftarrow} U(j)$. From (4.2) it follows that the identical mapping $U(\infty) \mapsto U_{\tau}(\infty)$ is continuous.

We equip every group $U(j)$ with the probability Haar measure $\mu_{j}$. As is well known [2], Theorem 1.6, the image measure $\pi_{j-1}^{j}\left(\mu_{j}\right)$ is equal to $\mu_{j-1}$. In other words, $\mu_{j-1}(\Omega)=$ $\left[\mu_{j} \circ\left(\pi_{j-1}^{j}\right)^{-1}\right](\Omega)$ for all Borel sets $\Omega$ in $U(j-1)$. Following [3], Lemma 4.8 and [2], n. 3.1, with the help of the Kolmogorov consistency theorem, we uniquely define on $\lim _{\leftarrow} U(j)$ the probability Radon measure $\overleftarrow{\mu}$ as the projective limit of the sequence $\left(\mu_{j}\right)$ under the mappings $\pi_{j-1}^{j}$ :

$$
\overleftarrow{\mu}:=\lim _{\longleftarrow} \mu_{j} \quad \text { so that } \quad \mu_{j}=\pi_{j}(\overleftarrow{\mu}) \quad \text { for all } j \in \mathbb{N}
$$

where the image $\pi_{j}(\overleftarrow{\mu})$ is such that $\mu_{j}(\Omega)=\left(\overleftarrow{\mu} \circ \pi_{j}^{-1}\right)(\Omega)$ for all Borel sets $\Omega$ in $U(j)$

Theorem 4.1 There exists a unique probability Radon measure $\mu$ on $U(\infty)$ such that $\overleftarrow{\mu}(\Omega)=\left(\mu \circ \tau^{-1}\right)(\Omega)$ for all Borel sets $\Omega \subset \lim U(j)$ and

$$
\int f(u \cdot g) d \mu(u)=\int f(u) d \mu(u), \quad g \in U^{2}(\infty), f \in C_{b}(U(\infty)),
$$

where $C_{b}(U(\infty))$ is the algebra of bounded continuous complex-valued functions on $U(\infty)$. Moreover, there exists a subsequence of Haar measures $\left(\mu_{j_{k}}\right)$ that weakly converges to $\mu$ in the sense that

$$
\lim _{k \rightarrow \infty} \int f d \mu_{j_{k}}=\int f d \mu \quad \text { for all } f \in C_{b}\left(U_{\tau}(\infty)\right)
$$

where $C_{b}\left(U_{\tau}(\infty)\right)$ is the subalgebra in $C_{b}(U(\infty))$ of continuous functions on $U_{\tau}(\infty)$.

Proof Let $\check{U}(j) \subset U(j)$ be the set of matrices for which $\{-1\}$ is not an eigenvalue. As is known [3], n. 3, $\breve{U}(j)$ is open in $U(j)$, and $\mu_{j}(U(j) \backslash \check{U}(j))=0$. In virtue of [3], Lemma 3.11, the restrictions $\pi_{j-1}^{j}: \breve{U}(j) \rightarrow \breve{U}(j-1)$ are continuous and surjective. Define the projective limit $\lim _{\leftarrow} \breve{U}(j)$ under these continuous mappings. Note that $\pi_{j}: \lim _{\leftarrow} \check{U}(j) \rightarrow \breve{U}(j)$ are also continuous and surjective. 
As is well known (see, e.g., [11], Theorem 6), by the Prokhorov criterion there exists a Radon probability measure $\check{\mu}$ on $\lim \check{U}(j)$ such that $\pi_{j}(\check{\mu})=\mu_{j}$ for all $j \in \mathbb{N}$ iff for every $\varepsilon>0$, there exists a compact set $\mathcal{K}$ in $\lim _{\longleftarrow} \breve{U}(j)$ such that $\left(\mu_{j} \circ \pi_{j}\right)(\mathcal{K}) \geq 1-\varepsilon$ for all $j \in \mathbb{N}$. In this case, $\breve{\mu}$ is uniquely determined by the formula

$$
\check{\mu}(\mathcal{K})=\inf _{j}\left(\mu_{j} \circ \pi_{j}\right)(\mathcal{K}) .
$$

Apply this criterion. Since $\mu_{k}(U(k) \backslash \check{U}(k))=0, \sup _{K_{k} \subset \check{U}(k)} \mu_{k}\left(K_{k}\right)=1$ as $K_{k}$ runs over all compact sets in $\check{U}(k)$. It follows that for every $\varepsilon>0$, there exists a compact set $K_{k} \subset \check{U}(k)$ such that

$$
\mu_{k}\left(K_{k}\right) \geq 1-\varepsilon
$$

In accordance with (4.2), we put $K_{j}:=\pi_{j}^{k}\left(K_{k}\right)$ for $j<k$ and $K_{j}:=\left[\begin{array}{cc}K_{k} & 0 \\ 0 & \mathbb{1}\end{array}\right]$ for $j \geq k$. Taking into account the definition of image measures, we have

$$
\mu_{j}\left(K_{j}\right)=\left\{\begin{array}{ll}
\mu_{k}\left(K_{k}\right)=\left[\mu_{k} \circ\left(\pi_{j}^{k}\right)^{-1}\right]\left(K_{j}\right): & j<k, \\
\mu_{k}\left(K_{k}\right): & j \geq k
\end{array} \quad \text { for all } j \in \mathbb{N} .\right.
$$

Thus, for any compact set $\mathcal{K}=\left(K_{j}\right) \subset \lim \check{U}(j)$ such that condition (4.5) for $K_{k}=\pi_{k}(\mathcal{K})$ with fixed $k$ is satisfied and $K_{j}=\pi_{j}(\mathcal{K})$ for all other $j \neq k$ are defined in accordance with (4.2), the following condition holds:

$$
\left(\mu_{j} \circ \pi_{j}\right)(\mathcal{K})=\mu_{k}\left(K_{k}\right) \geq 1-\varepsilon \quad \text { for all } j \in \mathbb{N} .
$$

So, the necessary and sufficient conditions of Prokhorov's criterion are satisfied. Thus, there exists a unique Radon probability measure $\check{\mu}$ on $\lim _{\longleftarrow} \check{U}(j)$ such that $\pi_{j}(\check{\mu})=\mu_{j}$ for all $j \in \mathbb{N}$ and

$$
\check{\mu}(\mathcal{K})=\inf _{j} \mu_{j}\left(K_{j}\right)=\mu_{k}\left(K_{k}\right)
$$

because of equalities (4.6). This measure $\check{\mu}$ can be extended to $\lim _{\leftarrow} U(j) \backslash \lim \check{L}(j)$ as zero since $\mu_{k}$ is zero on $U(k) \backslash \check{U}(k)$. Consequently, $\left.\check{\mu}(\mathcal{K} \cdot g)=\inf _{j} \mu_{j} \overleftarrow{\left(K_{j}\right.} \cdot g\right)=\mu_{k}\left(K_{k} \cdot g\right)$ for all $g \in U^{2}(k)$. The invariance property of the Haar measures $\mu_{k}$ yields

$$
\check{\mu}(\mathcal{K} \cdot g)=\mu_{k}\left(K_{k} \cdot g\right)=\mu_{k}\left(K_{k}\right)=\check{\mu}(\mathcal{K}) \quad \text { for all } g \in U^{2}(k) \text {. }
$$

Hence, $\check{\mu}$ is invariant under the right actions (see also [2], Proposition 3.2). It remains to note that the uniqueness property of the projective limit $\lim _{\leftarrow} \mu_{j}$ implies that $\check{\mu}=\overleftarrow{\mu}$.

The inductive limit $U_{\tau}(\infty)$ is regular because inclusions $U(j) \rightarrow U(j+1)$ are compact. Hence, any compact subset of $U_{\tau}(\infty)$ is contained in a subgroup $U(k)$ with fixed $k$. In virtue of (4.7) and the equality $\check{\mu}=\overleftarrow{\mu}$, we obtain

$$
\sup _{\mathcal{K}} \overleftarrow{\mu}(\mathcal{K})=1 \quad\left(\text { since } \sup _{K_{k} \subset U(k)} \mu_{k}\left(K_{k}\right)=1\right)
$$


where the supremum is taken over all compact sets $\mathcal{K}=\left(K_{j}\right)$ in $\lim U(j)$ such that $\tau^{-1}(\mathcal{K})$ coincides with $K_{k}=\pi_{k}(\mathcal{K})$. By the known Schwartz theorem (see, e.g., [11], Theorem 5) condition (4.9) is necessary and sufficient for the existence of a unique probability Radon measure $\mu$ on $U_{\tau}(\infty)$ such that $\overleftarrow{\mu}(\Omega)=\left(\mu \circ \tau^{-1}\right)(\Omega)$ for all Borel sets $\Omega \subset \lim _{\leftarrow} U(j)$. In other words, the measure $\overleftarrow{\mu}$ coincides with the image of $\mu$ under $\tau$, that is, $\overleftarrow{\mu}=\tau(\mu)$. By (4.8) and the equality $\check{\mu}=\overleftarrow{\mu}$,

$$
\mu(K \cdot g)=\mu(K) \quad \text { for all } K=\tau^{-1}(\Omega) \subset U(\infty), g \in U^{2}(\infty),
$$

which directly yields (4.3).

Let $C_{b}\left(U_{\tau}(\infty)\right)$ be endowed with the uniform norm. Since $U_{\tau}(\infty)$ is metric, the Prokhorov criterion provides the relative compactness property of the sequence $\left(\mu_{j}\right)$ in the dual space $C_{b}^{\prime}\left(U_{\tau}(\infty)\right)$ endowed with the weak topology. This gives the equality (4.4) since it holds over the dense subspace $C_{0}\left(U_{\tau}(\infty)\right)$ of functions with compact supports.

Corollary 4.2 The following integral formulas hold:

$$
\begin{aligned}
& \int f d \mu=\int d \mu(u) \int_{U^{2}(j)} f(u \cdot g) d\left(\mu_{j} \otimes \mu_{j}\right)(g), \\
& \int f d \mu=\frac{1}{2 \pi} \int d \mu(u) \int_{-\pi}^{\pi} f[\exp (\mathfrak{\mathbb { 1 } \vartheta}) u] d \vartheta, \quad f \in C_{b}(U(\infty)) .
\end{aligned}
$$

Proof Applying the invariance property (4.3) and the Fubini theorem, similarly to [12], Lemma 2, we get the integral formulas (4.10)-(4.11).

Consider a concentration property of a relatively compact sequence of Haar measures $\left(\mu_{j}\right)$ in the case where the corresponding group $U(j)$ is endowed with the normalized Hilbert-Schmidt metric

$$
d_{H S}(u, v)=\sqrt{j^{-1} \operatorname{tr}|u-v|_{H S}}, \quad \text { where }|u-v|_{H S}=\sqrt{(u-v)^{*}(u-v)} .
$$

This metric is a standard $\ell^{2}$-distance between matrices $u, v \in U(j)$, viewed as elements of a $j^{2}$-dimensional Hilbert space, which is normalized so as to make the identity $(j \times j)$-matrix have norm one. The bi-invariance $d_{H S}(u, v)=d_{H S}(u \cdot g, v \cdot g)$ for all $g \in U^{2}(j)$ is a consequence of the trace property $\operatorname{tr}(u v)=\operatorname{tr}(v u)$. We define the $\varepsilon$-neighborhood of $K_{j} \subset U(j)$ by

$$
\left(K_{j}\right)_{\varepsilon}:=\left\{u_{j} \in U(j): d_{H S}\left(u_{j}, K_{j}\right)<\varepsilon\right\}
$$

Theorem 4.3 For every $\varepsilon>0$ and closed set $K \subset U(\infty)$ such that $\mu_{j}\left(K_{j}\right) \geq 1 / 2$ where $K_{j}:=$ $K \cap U(j)$ for all $j \in \mathbb{N}$, the following equalities hold:

$$
\mu\left(K_{\varepsilon+\eta}\right)=\lim _{j \rightarrow \infty} \mu_{j}\left[\left(K_{j}\right)_{\varepsilon}\right]=1, \quad K_{\varepsilon+\eta}:=\bigcup\left(K_{j}\right)_{\varepsilon+\eta}, \quad \eta>0 .
$$

Proof As is well known (see [13]), $\left(U(j), d_{H S}, \mu_{j}\right)$ forms the Lévy sequence, that is, $\lim _{j \rightarrow \infty} \mu_{j}\left[\left(K_{j}\right)_{\varepsilon}\right]=1$ for any $\varepsilon>0$ and any closed set $K \subset U(\infty)$ such that $\mu_{j}\left(K_{j}\right) \geq 1 / 2$ 
for all $j \in \mathbb{N}$. The topological space $U_{\tau}(\infty)$ is completely regular. Hence, the closed set $K_{\varepsilon}=\overline{\bigcup\left(K_{j}\right)_{\varepsilon}}$ can be separated by a continuous function. Taking in (4.4) a function $f \in C_{b}\left(U_{\tau}(\infty)\right)$ such that $0 \leq f \leq 1$ where $\left.f\right|_{K_{\varepsilon}} \equiv 1$ and $\left.f\right|_{U(\infty) \backslash K_{\varepsilon+\eta}} \equiv 0$, we obtain

$$
\mu\left(K_{\varepsilon+\eta}\right) \geq \int f d \mu=\lim _{k \rightarrow \infty} \int f d \mu_{j_{k}} \geq \lim _{k \rightarrow \infty} \mu_{j_{k}}\left[\left(K_{j_{k}}\right)_{\varepsilon}\right]=1
$$

for a weakly convergent subsequence $\left(\mu_{j_{k}}\right)$. It follows that $\mu\left(K_{\varepsilon+\eta}\right)=1$ because $1=$ $\mu(U(\infty)) \geq \mu\left(K_{\varepsilon+\eta}\right)$.

\section{Hardy spaces $\mathcal{H}_{\mu}^{p}(1 \leq p \leq \infty)$}

In what follows, the space of complex functions $f$ on $U(\infty)$ endowed with the norm

$$
\|f\|_{L_{\mu}^{p}}= \begin{cases}\sqrt[p]{\int|f|^{p} d \mu}, & 1 \leq p<\infty, \\ {\operatorname{ess} \sup _{u \in U(\infty)}}|f(u)|, & p=\infty\end{cases}
$$

is denoted by $L_{\mu}^{p}$. It is clear that $L_{\mu}^{\infty} q L_{\mu}^{p}$ and $\|f\|_{L_{\mu}^{p}} \leq\|f\|_{L_{\mu}^{\infty}}$ for all $f \in L_{\mu}^{\infty}$.

We still assume that for any basis element $e_{j}$ in $H$, there exist $z_{j} \in E^{\prime}$ such that $J^{*} z_{j}=e_{j}$. By transitivity the orbits $\{u(e): u \in U(\infty)\} \subset S$ do not depend on the choice of $e \in S \cap \mathscr{R}\left(J^{*}\right)$. Fix an arbitrary $e \in S \cap \mathscr{R}\left(J^{*}\right)$.

To a pair $(\lambda, l) \in \mathbb{Y} \times \mathbb{N}_{*}^{\ell(\lambda)}$, we assign the $\ell(\lambda)$-dimensional complex subspace $H_{l}=$ $\operatorname{span}\left\{e_{l_{1}}, \ldots, e_{l_{\ell(\lambda)}}\right\}$. On the dense subspace $\bigcup H_{l}$ in $H$ there is well defined the $C_{b}(U(\infty))$ valued linear mapping

$$
\phi: h \mapsto \phi_{h}(u)=\langle u(e) \mid h\rangle, \quad u \in U(\infty) .
$$

It can be shown that $\phi$ may be isometrically extended onto $H$ as an $L_{\mu}^{2}$-valued mapping, which is still defined on $E^{\prime}$ as $\phi \circ A$.

Remark 5.1 Note that in the case of a Gaussian measure $\mu$ on $E$ there exists a unique extension $\phi: h \mapsto\langle\cdot \mid h\rangle$ from $\mathscr{R}\left(J^{*}\right)$ to the isometric embedding $H \rightarrow L_{\mu}^{2}$, which is called the Paley-Wiener map (see, e.g., [14]).

By the polarization formula for symmetric tensor products, to every $e_{l}^{\odot \lambda} \in e^{\odot \mathbb{Y}}$ there uniquely corresponds the function

$$
\phi_{l}^{\lambda}(u):=\prod_{k=1}^{\ell(\lambda)} \phi_{e_{l_{k}}}^{\lambda_{k}}(u)=\left\langle[u(e)]^{\otimes|\lambda|} \mid e_{l}^{\odot \lambda}\right\rangle, \quad \phi_{e_{l_{k}}}(u)=\left\langle u(e) \mid e_{l_{k}}\right\rangle,
$$

belonging to $C_{b}(U(\infty))$ in the variable $u \in U(\infty)$, where $\phi_{l_{k}}:=\phi_{e_{l_{k}}}$.

We define the Hardy space $\mathcal{H}_{\mu}^{p}(1 \leq p \leq \infty)$ with respect to the Wiener measure $\mu$ associated with the covariance operator $J \circ J^{*}$ (resp., its subspace $\mathcal{H}_{\mu}^{p, n}$ with a fixed $n \in \mathbb{Z}_{+}$) to be the $L_{\mu}^{p}$-closed complex linear span of the system

$$
\phi^{\mathbb{Y}}=\left\{\phi_{l}^{\lambda}:(\lambda, l) \in \mathbb{Y} \times \mathbb{N}_{*}^{\ell(\lambda)}\right\} \quad\left(\text { resp. }, \phi^{\mathbb{Y}_{n}}=\left\{\phi_{l}^{\lambda} \in \phi^{\mathbb{Y}}:(\lambda, l) \in \mathbb{Y}_{n} \times \mathbb{N}_{*}^{\ell(\lambda)}\right\}\right),
$$

where $\phi_{l}^{\emptyset} \equiv 1$. The following theorem for a different case is proved in [12], Theorem 6 . 
Theorem 5.1 The system $\phi^{\mathbb{Y}}$ is orthogonal in $L_{\mu}^{2}$, and

$$
\left\|\phi_{l}^{\lambda}\right\|_{L_{\mu}^{2}}^{2}=\frac{(\ell(\lambda)-1) ! \lambda !}{(\ell(\lambda)-1+|\lambda|) !}, \quad(\lambda, l) \in \mathbb{Y} \times \mathbb{N}_{*}^{\ell(\lambda)} .
$$

Proof The orthogonal property $\phi_{j}^{\lambda^{\prime}} \perp \phi_{l}^{\lambda}$ with $\left|\lambda^{\prime}\right| \neq|\lambda|$ follows from (4.11) since

$$
\int \phi_{J}^{\lambda^{\prime}} \bar{\phi}_{l}^{\lambda} d \mu=\frac{1}{2 \pi} \int \phi_{J}^{\lambda^{\prime}} \bar{\phi}_{l}^{\lambda} d \mu \int_{-\pi}^{\pi} \exp \left[\stackrel{i}{1}\left(\left|\lambda^{\prime}\right|-|\lambda|\right) \vartheta\right] d \vartheta=0
$$

for any $\lambda^{\prime}, \lambda \in \mathbb{Y} \backslash\{\emptyset\}$. Let $\left|\lambda^{\prime}\right|=|\lambda|$ and $\ell\left(\lambda^{\prime}\right)>\ell(\lambda)$ for definiteness. Then there exists an index $k$ with an appropriate nonzero integer $\lambda_{k}^{\prime}$ in the diagram $\lambda^{\prime}=\left(\lambda_{1}^{\prime}, \ldots, \lambda_{k}^{\prime}, \ldots, \lambda_{\ell\left(\lambda^{\prime}\right)}^{\prime}\right) \in$ $\mathbb{Y} \backslash\{\emptyset\}$ such that $\ell(\lambda)<k \leq \ell\left(\lambda^{\prime}\right)$. In this case, we have $\phi_{j}^{\lambda^{\prime}} \perp \phi_{l}^{\lambda}$ because formula (4.11) implies

$$
\int \phi_{J}^{\lambda^{\prime}} \bar{\phi}_{l}^{\lambda} d \mu=\frac{1}{2 \pi} \int \phi_{j}^{\lambda^{\prime}} \bar{\phi}_{l}^{\lambda} d \mu \int_{-\pi}^{\pi} \exp \left(i \lambda_{k}^{\prime} \vartheta\right) d \vartheta=0
$$

Consider the case $\left|\lambda^{\prime}\right|=|\lambda|$ and $\ell\left(\lambda^{\prime}\right)=\ell(\lambda)$. If $\phi_{j}^{\lambda^{\prime}} \neq \phi_{l}^{\lambda}$, then $\lambda^{\prime} \neq \lambda$. There exists an index $0<k \leq \ell(\lambda)$ such that $\lambda_{k}^{\prime} \neq \lambda_{k}$. Similarly as before, $\phi_{j}^{\lambda^{\prime}} \perp \phi_{l}^{\lambda}$ because

$$
\left.\int \phi_{J}^{\lambda^{\prime}} \bar{\phi}_{l}^{\lambda} d \mu=\frac{1}{2 \pi} \int \phi_{J}^{\lambda^{\prime}} \bar{\phi}_{l}^{\lambda} d \mu \int_{-\pi}^{\pi} \exp \left[\stackrel{\check{1}}{(} \lambda_{k}^{\prime}-\lambda_{k}\right) \vartheta\right] d \vartheta=0
$$

Let $H_{l}$ with $\imath=\left(l_{1}, \ldots, l_{\ell(\lambda)}\right) \in \mathbb{N}_{*}^{\ell(\lambda)}$ be the $\ell(\lambda)$-dimensional subspace in $H$ spanned by $\left\{e_{l_{1}}, \ldots, e_{l_{\ell(\lambda)}}\right\}$, and $U(\imath)$ be the unitary subgroup of $U(\infty)$ acting in $H_{l}$. Let $g_{l}=\left(\mathbb{1}_{l}, w_{l}\right) \in$ $U^{2}(\imath)$. Using (4.10) with $U(l)$ instead of $U(j)$ recursively by $k=1, \ldots, \ell(\lambda)$, we get

$$
\int\left|\phi_{l}^{\lambda}\right|^{2} d \mu=\int d \mu(u) \prod_{k=1}^{\ell(\lambda)} \int_{U(l)}\left|\left\langle w_{l}^{-1} u(e) \mid e_{l_{k}}\right\rangle\right|^{2} d \mu_{l}\left(w_{l}\right) .
$$

Integrals with the Haar measures $\mu_{\iota}$ are independent of $u \in U(\infty)$. Hence,

$$
\int_{U(l)}\left|\left\langle w_{l}^{-1} u(e) \mid e_{l_{k}}\right\rangle\right|^{2} d \mu_{l}\left(w_{l}\right)=\frac{(\ell(\lambda)-1) ! \lambda !}{(\ell(\lambda)-1+|\lambda|) !}
$$

by the well-known integral formula for unitary groups [15], n. 1.4.9. It remains to note that the last formulas immediately yield (5.3) because $\int d \mu=1$.

Theorem 5.1 directly implies that $\phi$ has an isometric extension onto $H$ and that the following orthogonal expansion holds:

$$
\mathcal{H}_{\mu}^{2}=\mathbb{C} \oplus \mathcal{H}_{\mu}^{2,1} \oplus \mathcal{H}_{\mu}^{2,2} \oplus \cdots
$$

Remark 5.2 In the case of a Gaussian measure $\mu$ on $E$, decomposition (5.4) is called the Wiener-Itô chaos expansion. 


\section{Inverse integral formulas}

The correspondence $e_{l}^{\odot \lambda} \mapsto \phi_{l}^{\lambda}$ allows us to define a conjugate-linear isomorphism $\Gamma \rightarrow$ $\mathcal{H}_{\mu}^{2}$. As a result, the linear isometry $\Phi: \mathcal{H}^{2} \rightarrow \mathcal{H}_{\mu}^{2}$ and its adjoint $\Phi^{*}: \mathcal{H}_{\mu}^{2} \rightarrow \mathcal{H}^{2}$ can be uniquely defined by the change of orthonormal bases

$$
\Phi: \mathcal{H}^{2} \ni \zeta_{l}^{\lambda}\left\|e_{l}^{\odot \lambda}\right\|_{\Gamma}^{-1} \mapsto \phi_{l}^{\lambda}\left\|\phi_{l}^{\lambda}\right\|_{L_{\mu}^{2}}^{-1} \in \mathcal{H}_{\mu}^{2}, \quad \lambda \in \mathbb{Y}, \imath \in \mathbb{N}_{*}^{\ell(\lambda)} .
$$

Clearly, $\Phi^{*}: \phi_{l}^{\lambda}\left\|\phi_{l}^{\lambda}\right\|_{L_{\mu}^{2}}^{-1} \mapsto \zeta_{l}^{\lambda}\left\|e_{l}^{\odot \lambda}\right\|_{\Gamma}^{-1}$ since $\left\langle\Phi \zeta_{l}^{\lambda} \mid f\right\rangle_{L_{\mu}^{2}}=\left\langle\zeta_{l}^{\lambda} \mid \Phi^{*} f\right\rangle_{\mathcal{H}^{2}}$ for all $f \in \mathcal{H}_{\mu}^{2}$. Hence, for any $\psi^{\star} \in \mathcal{H}^{2}$ with the Fourier coefficients $\tilde{\psi}^{\star}(\lambda, l)$ defined in (3.2), we obtain

$$
\Phi \psi^{\star}=\sum_{(\lambda, l) \in \mathbb{Y} \times \mathbb{N}_{*}^{\ell(\lambda)}} \tilde{\psi}^{\star}(\lambda, l) \frac{\left\|e_{l}^{\odot \lambda}\right\|_{\Gamma}^{2}}{\left\|\phi_{l}^{\lambda}\right\|_{L_{\mu}^{2}}^{2}} \phi_{l}^{\lambda}, \quad \text { where } \frac{\left\|e_{l}^{\odot \lambda}\right\|_{\Gamma}^{2}}{\left\|\phi_{l}^{\lambda}\right\|_{L_{\mu}^{2}}^{2}}=\frac{(\ell(\lambda)-1+|\lambda|) !}{(\ell(\lambda)-1) !|\lambda| !} .
$$

In particular, $\phi_{J^{*} z}=\sum \bar{\zeta}_{j}(z) \phi_{e_{j}}$ and $\left\|\phi_{J^{*} z}\right\|_{L_{\mu}^{2}}^{2}=\sum\left|\zeta_{j}(z)\right|^{2}=\|z\|_{J^{*}}^{2}$ for any $z \in E^{\prime}$. Hence, if $E^{\prime}$ is endowed with the norm $\|\cdot\|_{J^{*}}$, then the embedding

$$
\phi \circ A:\left(E^{\prime},\|\cdot\|_{J^{*}}\right) \ni z \mapsto \phi_{J^{*} z} \in L_{\mu}^{2}
$$

is the isometric extension of (5.1), and its image coincides with the subspace $\mathcal{H}_{\mu}^{2,1}$.

We call the isometric embedding (6.1) the Paley-Wiener map corresponding to $\mu$.

Thus, the mapping $\Phi$ is an isometric extension of the Paley-Wiener map $\phi \circ A$ since $\left.\Phi\right|_{E^{\prime}}=\phi \circ A$.

Lemma 6.1 The vector-valued functions with respect to the variable $u \in U(\infty), \mathcal{Q} \ni z \mapsto$ $(\Phi \circ \mathcal{C})(u, z)$ and $\mathcal{Q} \ni z \mapsto(\Phi \circ \mathcal{P})(u, z)$, take values in the space $L_{\mu}^{\infty}$ and may be written as follows:

$$
(\Phi \circ \mathcal{C})(u, z)=\frac{1}{1-\phi_{J^{*} z}(u)}, \quad(\Phi \circ \mathcal{P})(u, z)=\frac{1-\|z\|_{J^{*}}^{2}}{\left|1-\phi_{J^{*} z}(u)\right|^{2}} .
$$

Proof Let $h=J^{*} z$. The Fourier decomposition $h=\sum \zeta_{j}(z) e_{j}$ yields $\phi_{h}=\sum \bar{\zeta}_{j}(z) \phi_{e_{j}}$. Applying $\Phi$ to the Fourier decomposition of $\mathcal{C}\left(z^{\prime}, z\right)$ under the variable $z^{\prime} \in \mathcal{Q}$, we obtain

$$
(\Phi \circ \mathcal{C})(u, z)=\sum_{(\lambda, l)} \frac{\bar{\zeta}_{l}^{\lambda}(z) \phi_{l}^{\lambda}(u)}{\left\|e_{l}^{\odot \lambda}\right\|_{\Gamma}^{2}}=\sum_{n \in \mathbb{Z}_{+}}\left(\sum_{j \in \mathbb{N}} \bar{\zeta}_{j}(z) \phi_{e_{j}}(u)\right)^{n}=\frac{1}{1-\phi_{h}(u)}
$$

because $\left\|e_{t}^{\odot \lambda}\right\|_{\Gamma}^{-2}=n ! / \lambda$ ! coincide with multinomial coefficients. It follows that $\mid(\Phi \circ$ $\mathcal{C})(u, z) \mid \leq\left(1-\left|\phi_{h}\right|\right)^{-1}<\infty$ for all $z \in \mathcal{Q}$.

Similarly, applying $\Phi$ to the Fourier decomposition of $\mathcal{P}(\cdot, z)$, we obtain

$$
(\Phi \circ \mathcal{P})(u, z)=\left|\sum_{(\lambda, l)} \frac{\bar{\zeta}_{l}^{\lambda}(z) \phi_{l}^{\lambda}(u)}{\left\|e_{l}^{\odot \lambda}\right\|_{\Gamma}^{2}}\right|^{2}\left(\sum_{(\lambda, l)} \frac{\left|\zeta_{l}^{\lambda}(z)\right|^{2}}{\left\|e_{l}^{\odot \lambda}\right\|_{\Gamma}^{2}}\right)^{-1}=\frac{1-\|z\|_{J^{*}}^{2}}{\left|1-\phi_{h}(u)\right|^{2}} .
$$

Again using Theorem 5.1, we get

$$
(\Phi \circ \mathcal{P})(u, z)=\frac{1-\|z\|_{J^{*}}^{2}}{\left|1-\phi_{h}(u)\right|^{2}} \leq\left(1-\|z\|_{J^{*}}^{2}\right)\left(\sum_{n \in \mathbb{Z}_{+}}\|z\|_{J^{*}}^{n}\right)^{2}=\frac{1-\|z\|_{J^{*}}}{\left(1-\|z\|_{J^{*}}\right)^{2}}=\frac{1+\|z\|_{J^{*}}}{1-\|z\|_{J^{*}}} .
$$

As a result, $(\Phi \circ \mathcal{C})(\cdot, z)$ and $(\Phi \circ \mathcal{P})(\cdot, z)$ with $z \in \mathcal{Q}$ take values in $L_{\mu}^{\infty}$. 
Theorem 6.2 For any $f \in \mathcal{H}_{\mu}^{2}$, the function

$$
\mathcal{C}[f](z):=\left\langle\left(\Phi^{*} \circ f\right)(\cdot)|\mathcal{C}(\cdot, z)|_{\mathcal{H}^{2}}=\left\langle\left(\Phi^{*} \circ f\right)(\cdot)|\mathcal{P}(\cdot, z)|_{\mathcal{H}^{2}}, \quad z \in \mathcal{Q},\right.\right.
$$

belongs to the space of analytic functions $\mathcal{H}^{2}$ and has the integral representations

$$
\mathcal{C}[f](z)=\int \frac{f d \mu}{1-\bar{\phi}_{J^{*} z}}=\int \frac{1-\|z\|_{J^{*}}^{2}}{\left|1-\bar{\phi}_{J^{*} z}(u)\right|^{2}} f(u) d \mu(u) .
$$

The mapping $f \mapsto \mathcal{C}[f]$ generated by $\Phi^{*}$ produces the isometry $\mathcal{H}_{\mu}^{2} \simeq \mathcal{H}^{2}$.

Proof Consider the orthogonal decomposition with respect to $\phi^{\mathbb{Y}}$ and its $\Phi^{*}$-image

$$
f=\sum_{(\lambda, l) \in \mathbb{Y} \times \mathbb{N}_{*}^{\ell(\lambda)}} \tilde{f}(\lambda, l) \phi_{l}^{\lambda}, \quad \Phi^{*} f=\sum_{(\lambda, l) \in \mathbb{Y} \times \mathbb{N}_{*}^{\ell(\lambda)}} \tilde{f}(\lambda, l) \frac{\left\|\phi_{l}^{\lambda}\right\|_{L_{\mu}^{2}}^{2}}{\left\|e_{l}^{\odot \lambda}\right\|_{\Gamma}^{2}} \zeta_{l}^{\lambda},
$$

respectively, where $\tilde{f}(\lambda, l):=\left\|\phi_{l}^{\lambda}\right\|_{L_{\mu}^{2}}^{-2} \int f \bar{\phi}_{l}^{\lambda} d \mu$ are the Fourier coefficients. Substituting their to $\mathcal{C}[f]$ and taking into account Lemma 6.1 together with orthogonal properties, we get the first equality in (6.3)

$$
\begin{aligned}
\mathcal{C}[f](z) & =\sum_{(\lambda, l)} \frac{\tilde{f}(\lambda, l) \zeta_{l}^{\lambda}(z)\left\|\phi_{l}^{\lambda}\right\|_{L_{\mu}^{2}}^{2}\left\langle\zeta_{l}^{\lambda} \mid \zeta_{l}^{\lambda}\right\rangle_{\mathcal{H}^{2}}}{\left\|e_{l}^{\odot \lambda}\right\|_{\Gamma}^{4}} \\
& =\int \sum_{(\lambda, l)} \frac{\zeta_{l}^{\lambda}(z) \bar{\phi}_{l}^{\lambda}}{\left\|e_{l}^{\odot \lambda}\right\|_{\Gamma}^{2}} f d \mu=\int(\Phi \circ \mathcal{C})(\cdot, z) f d \mu=\int \frac{f d \mu}{1-\bar{\phi}_{J^{*} z}} .
\end{aligned}
$$

To check the second equality in (6.3), we also apply Lemma 6.1. As a result,

$$
\begin{aligned}
\mathcal{C}[f](z) & =\left\langle\left(\Phi^{*} \circ f\right)(\cdot)|\mathcal{P}(\cdot, z)|_{\mathcal{H}^{2}}\right. \\
& =\int(\Phi \circ \mathcal{P})(z, \cdot) f d \mu=\int \frac{1-\|z\|_{J^{*}}^{2}}{\left|1-\bar{\phi}_{J^{*} z}(u)\right|^{2}} f(u) d \mu(u) .
\end{aligned}
$$

Hence, both integral representations in (6.3) hold. Since $\mathscr{R}\left(\Phi^{*}\right)=\mathcal{H}^{2}$, Lemma 3.2 implies that the mapping $\Phi^{*}: \mathcal{H}_{\mu}^{2} \ni f \mapsto \mathcal{C}[f] \in \mathcal{H}^{2}$ is surjective.

Remark 6.1 The $L_{\mu}^{\infty}$-valued function $\mathcal{Q} \ni z \mapsto(\Phi \circ \mathcal{P})(\cdot, z)$ is a Poisson-type kernel for the infinite-dimensional ball $\mathcal{Q}$. The second integral formula in (6.3) is a Poisson-type formula over the Hardy space $\mathcal{H}_{\mu}^{2}$.

Remark 6.2 Since $\Phi^{*}: \mathcal{H}_{\mu}^{2} \ni f \mapsto \mathcal{C}[f] \in \mathcal{H}^{2}$ is isometric and surjective, the integral formulas (6.3) are inverse to the transform $\Phi$, which is an isometric extension of the PaleyWiener map $\phi \circ A$.

\section{Directional derivatives}

Now we calculate the directional derivatives of an analytic function $\psi^{\star} \in \mathcal{H}^{2}$ at any point $z \in \mathcal{Q}$ :

$$
\mathfrak{d}_{a} \psi^{\star}(z):=\lim _{t \rightarrow 0} \frac{\psi^{\star}(z+t a)-\psi^{\star}(z)}{t}=\left.\frac{d \psi^{\star}(z+t a)}{d t}\right|_{t=0}, \quad a \in \mathcal{Q}, t \in \mathbb{R} .
$$


Consider the projector $S_{1} \otimes S_{n-1}: H^{\otimes n} \rightarrow H \otimes H^{\odot(n-1)}$ and its restriction $S_{n / 1}:=$ $\left.S_{n}\right|_{H \otimes H^{\odot(n-1)}}$ defined as $\eta \odot \psi_{n-1}=S_{n / 1}\left(\eta \otimes \psi_{n-1}\right) \in H^{\odot n}$ for all $\eta \in H$ and $\psi_{n-1} \in H^{\odot(n-1)}$. The projector $S_{n}$ possesses the decomposition $S_{n}=S_{n / 1} \circ\left(S_{1} \otimes S_{n-1}\right)$. For any $\lambda \in \mathbb{Y}$ such that $|\lambda|=n-1$ and $\imath \in \mathbb{N}^{\ell(\lambda)}$,

$$
\frac{1}{n}\left\|e_{m} \otimes e_{l}^{\odot \lambda}\right\|^{2}=\frac{1}{n} \frac{(\lambda) !}{(n-1) !}=\frac{(\lambda) !}{n !}=\left\|S_{n / 1}\left(e_{m} \otimes e_{l}^{\odot \lambda}\right)\right\|^{2}, \quad \text { so }\left\|S_{n / 1}\right\|=\frac{1}{n} .
$$

In fact, it suffices to decompose an element of $H \otimes H^{\odot(n-1)}$ with respect to the basis elements $e_{m} \otimes e_{l}^{\odot \lambda}$.

Define the operator $\delta_{a, n}: H^{\odot(n-1)} \rightarrow H^{\odot n}$ for a nonzero $a \in \mathcal{Q}$ as

$$
\begin{aligned}
\delta_{a, n}\left(J^{*} z\right)^{\otimes(n-1)}: & =n S_{n / 1}\left[J^{*} a \otimes\left(J^{*} z\right)^{\otimes(n-1)}\right] \\
& =\left.\frac{d\left(J^{*} z+t J^{*} a\right)^{\otimes n}}{d t}\right|_{t=0}=n J^{*} a \odot\left(J^{*} z\right)^{\otimes(n-1)},
\end{aligned}
$$

where the last equality is a consequence of the well-known tensor binomial formula $(x+$ $t y)^{\otimes n}=\sum_{m=0}^{n}\left(\begin{array}{c}m \\ n\end{array}\right)(t y)^{\otimes m} \odot x^{\otimes(n-m)}$ with any $x, y \in H$. Summing over $n \geq 1$, we define

$$
\delta_{a}\left(1-J^{*} z\right)^{-\otimes 1}:=\left.\bigoplus_{n \geq 1} \frac{d\left(J^{*} z+t J^{*} a\right)^{\otimes n}}{d t}\right|_{t=0}=\bigoplus_{n \geq 1} n J^{*} a \odot\left(J^{*} z\right)^{\otimes(n-1)} .
$$

Taking into account that $\left\|S_{n / 1}\right\|=n^{-1}$, we obtain

$$
\begin{aligned}
\left\|\delta_{a}\left(1-J^{*} z\right)^{-\otimes 1}\right\|_{\Gamma}^{2} & =\sum_{n \geq 1}\left\|n J^{*} a \odot\left(J^{*} z\right)^{\otimes(n-1)}\right\|_{\Gamma}^{2} \\
& \leq\|a\|_{J^{*}}^{2} \sum_{n \geq 1}\|z\|_{J^{*}}^{2(n-1)}=\|a\|_{J^{*}}^{2}\left\|\left(1-J^{*} z\right)^{-\otimes 1}\right\|_{\Gamma^{*}}^{2}
\end{aligned}
$$

Inequality (7.1) and the totality of $\left\{\left(1-J^{*} z\right)^{-\otimes 1}: z \in \mathcal{Q}\right\}$ in $\Gamma$ imply that the adjoint operator $\delta_{z}^{*}$ of $\delta_{z}$ on $\Gamma$ can be defined as $\delta_{z}^{*} \psi=\bigoplus_{n \geq 1} \delta_{z, n}^{*} \psi_{n}$. Here $\delta_{z, n}^{*}: H^{\odot n} \ni \psi_{n} \rightarrow \delta_{z, n}^{*} \psi_{n} \in H^{\odot(n-1)}$ is defined as the adjoint operator $\delta_{z, n}^{*}$ of $\delta_{z, n}$ on $H^{\otimes n}$ via the equality

$$
\left\langle\delta_{z, n}\left(J^{*} z\right)^{\otimes(n-1)} \mid \psi_{n}\right\rangle=\left\langle\left(J^{*} z\right)^{\otimes(n-1)} \mid \delta_{z, n}^{*} \psi_{n}\right\rangle .
$$

In fact, the image of $J^{*}$ contains all elements $\left(e_{m}\right)$; hence, $\left\{\left(J^{*} z\right)^{\otimes(n-1)}: z \in \mathcal{Q}\right\}$ is total in $H^{\odot(n-1)}$. So, by Riesz's theorem there exists unique $\delta_{z, n}^{*} \psi_{n} \in H^{\odot(n-1)}$, and $\delta_{z, n}^{*}$ is well defined.

As a consequence, from (7.1) we get $\left\|\delta_{a}^{*} \psi\right\|_{\Gamma} \leq\|a\|_{J^{*}}\|\psi\|_{\Gamma}$ for all $a \in \mathcal{Q}$ and $\psi \in \Gamma$, which means that $\delta_{a}^{*} \psi \in \Gamma$. So we have proved the following statement.

Lemma 7.1 For any function $\psi^{\star} \in \mathcal{H}^{2}$ associated with an element $\psi \in \Gamma$, we have that $\mathfrak{d}_{a} \psi^{\star} \in \mathcal{H}^{2}$ and $\mathfrak{d}_{a} \psi^{\star}(z)=\left\langle\left(1-J^{*} z\right)^{-\otimes 1} \mid \delta_{a}^{*} \psi\right\rangle$ for all $a, z \in \mathcal{Q}$.

Theorem 7.2 For any function $f \in \mathcal{H}_{\mu}^{2}$, we have $\mathfrak{d}_{a} \mathcal{C}[f] \in \mathcal{H}^{2}$, and the following formula holds:

$$
\mathfrak{d}_{a} \mathcal{C}[f](z)=\int \frac{f(u) \bar{\phi}_{J^{*} a}(u) d \mu(u)}{\left(1-\bar{\phi}_{J^{*} z}(u)\right)^{2}}, \quad a, z \in \mathcal{Q} .
$$


Proof First, note that $f \phi_{J^{*} a} \in \mathcal{H}_{\mu}^{2}$ for all $a \in \mathcal{Q}$ because $\phi_{J^{*} a} \in \mathcal{H}_{\mu}^{\infty}$. Moreover, $\mathfrak{d}_{a} \mathcal{C}[f] \in \mathcal{H}^{2}$ by Lemma 7.1. Using the first integral formula (6.3), we can write that

$$
\begin{aligned}
\mathfrak{d}_{a} \mathcal{C}[f](z) & =\left.\frac{d \mathcal{C}[f](z+t a)}{d t}\right|_{t=0} \\
& =\lim _{t \rightarrow 0} \frac{1}{t} \int\left(\frac{f(u)}{1-\bar{\phi}_{J^{*}(z+t a)}(u)}-\frac{f(u)}{1-\bar{\phi}_{J^{*} z}(u)}\right) d \mu(u) \\
& =\lim _{t \rightarrow 0} \frac{1}{t} \int\left(\frac{f(u)}{1-\left\langle J^{*}(z+t a) \mid u(e)\right\rangle}-\frac{f(u)}{1-\left\langle J^{*} z \mid u(e)\right\rangle}\right) d \mu(u) \\
& =\lim _{t \rightarrow 0} \frac{1}{t} \int \frac{t\left\langle J^{*} a \mid u(e)\right\rangle f(u) d \mu(u)}{\left(1-\left\langle J^{*}(z+t a) \mid u(e)\right\rangle\right)\left(1-\left\langle J^{*} z \mid u(e)\right\rangle\right)} \\
& =\lim _{t \rightarrow 0} \int \frac{\bar{\phi}_{J^{*} a}(u) f(u) d \mu(u)}{\left(1-\bar{\phi}_{J^{*}(z+t a)}(u)\right)\left(1-\bar{\phi}_{J^{*} z}(u)\right)}
\end{aligned}
$$

Now we need to prove that, as $t \rightarrow 0$,

$$
\begin{aligned}
& \int \frac{\bar{\phi}_{J^{*} a}(u) f(u) d \mu(u)}{\left(1-\bar{\phi}_{J^{*}(z+t a)}(u)\right)\left(1-\bar{\phi}_{J^{*} z}(u)\right)}-\int \frac{f(u) \bar{\phi}_{J^{*} a}(u) d \mu(u)}{\left(1-\bar{\phi}_{J^{*} z}(u)\right)^{2}} \\
& \quad=\int \frac{t \bar{\phi}_{J^{*} a}^{2}(u) f(u) d \mu(u)}{\left(1-\bar{\phi}_{J^{*}(z+t a)}(u)\right)\left(1-\bar{\phi}_{J^{*} z}(u)\right)^{2}} \rightarrow 0 .
\end{aligned}
$$

For a fixed $z \in \mathcal{Q}$, we put $\alpha:=\min \left\{\left|1-\bar{\phi}_{J^{*} z}(u)\right|: u \in U(\infty)\right\}$, so $\left|1-\bar{\phi}_{J^{*} z}(u)\right|^{2}>\alpha^{2}$,

$$
\alpha \leq\left|1-\bar{\phi}_{J^{*} z}(u)\right| \leq\left|1-\bar{\phi}_{J^{*}(z+t a)}(u)\right|+\left|t \bar{\phi}_{J^{*} a}(u)\right| .
$$

This yields $\left|1-\bar{\phi}_{J^{*}(z+t a)}(u)\right| \geq \alpha-\left|t \bar{\phi}_{J^{*} a}(u)\right| \geq \alpha / 2$ for $\left|t \bar{\phi}_{J^{*} a}(u)\right| \leq \alpha / 2$. It follows that

$$
\left|\int \frac{t \bar{\phi}_{J^{*} a}^{2}(u) f(u) d \mu(u)}{\left(1-\bar{\phi}_{J^{*}(z+t a)}(u)\right)\left(1-\bar{\phi}_{J^{*} z}(u)\right)^{2}}\right| \leq \frac{|t|}{\alpha / 2 \cdot \alpha^{2}} \int|f| d \mu \leq \frac{|t|}{\alpha / 2 \cdot \alpha^{2}}\|f\|_{L_{\mu}^{2}} \rightarrow 0
$$

as $t \rightarrow 0$. Hence, the integral formula (7.2) holds.

\section{Radial boundary values}

Set $J^{*} z=r v(e)$ with $z \in \mathcal{Q}, 0 \leq r<1$, and $v \in U(\infty)$, where $e \in S \cap \mathscr{R}\left(J^{*}\right)$ is a fixed element. Note that the corresponding complex-valued function

$$
U(\infty) \ni u \mapsto \phi_{J^{*} z}(u)=\langle u(e) \mid r v(e)\rangle
$$

satisfies the equalities $\phi_{J^{*} z}(u)=\phi_{r v(e)}(u)=r \phi_{\nu(e)}(u)=r \phi_{e}\left(v^{-1} u\right)$ where $v^{-1} u=u \cdot g$ is defined as the right action with $g=(\mathbb{1}, v) \in U^{2}(\infty)$. In particular, $\phi_{e}(\mathbb{1})=1$.

We define the Poisson kernel as follows:

$$
\mathcal{P}_{r}(v, u):=\frac{1-r^{2}}{\left|1-r \bar{\phi}_{e}\left(v^{-1} u\right)\right|^{2}}, \quad v, u \in U(\infty), 0 \leq r<1 .
$$

The Poisson integral is defined for any function $f \in \mathcal{H}_{\mu}^{p}(1 \leq p \leq \infty)$ as

$$
\mathcal{P}_{r}[f](v):=\int \mathcal{P}_{r}(v, u) f(u) d \mu(u), \quad v \in U(\infty), 0 \leq r<1 .
$$


It is easy to see that $\mathcal{P}_{r}[\operatorname{Re} f]=\operatorname{Re} \mathcal{P}_{r}[f]$ for all $f \in \mathcal{H}_{\mu}^{p}$. The following statement is an extension of Theorem 6.2 to the Hardy space $\mathcal{H}_{\mu}^{p}$ with an arbitrary $1 \leq p \leq \infty$.

Theorem 8.1 For every function $f \in \mathcal{H}_{\mu}^{p}(1 \leq p \leq \infty)$, the equalities

$$
\mathcal{P}_{r}[f](v)=\int \frac{f d \mu}{1-\bar{\phi}_{J^{*} z}}=\int \frac{1-\|z\|_{J^{*}}^{2}}{\left|1-\phi_{J^{*} z}\right|^{2}} f d \mu, \quad z=r A v(e) \in \mathcal{Q},
$$

hold, where the integrals are analytic in the variable $z \in \mathcal{Q}$.

Proof The space $\mathcal{H}_{\mu}^{p}$ is defined as the $L_{\mu}^{p}$-closed linear span of the orthogonal system $\phi^{\mathbb{Y}}$. On the other hand, the kernel $\mathcal{P}_{r}$ is related to the kernel $\Phi \circ \mathcal{P}$ in (6.2) by the equalities

$$
\mathcal{P}_{r}(v, \cdot)=(\Phi \circ \mathcal{P})(z, \cdot)=\frac{1-\|z\|_{J^{*}}^{2}}{\left|1-\phi_{J^{*} z}(\cdot)\right|^{2}}, \quad z=r A v(e) \in \mathcal{Q},
$$

where $\Phi \circ \mathcal{P}$ is an $L_{\mu}^{\infty}$-valued function in the variable $z$ via Lemma 6.1. Therefore, equalities (8.1) hold for any $f \in \mathcal{H}_{\mu}^{p}$ by orthogonality. The $L_{\mu}^{\infty}$-valued function $\mathcal{Q} \ni z \mapsto\left(1-\bar{\phi}_{J^{*} z}\right)^{-1}$ is analytic. Hence, the first integral in (8.1) is a complex-valued analytic function in the variable $z \in \mathcal{Q}$ as the composition of this $L_{\mu}^{\infty}$-valued function and the bounded linear functional $L_{\mu}^{\infty} \ni g \mapsto \int g f d \mu$ with $f \in \mathcal{H}_{\mu}^{p}$ because the embedding $L_{\mu}^{\infty} \rightarrow L_{\mu}^{p}(1 \leq p \leq \infty)$ is continuous.

Lemma 8.2 For any $u, v \in U(\infty)$ and $0 \leq r<1$, the kernel $\mathcal{P}_{r}$ satisfies the conditions

$$
\mathcal{P}_{r}(u, v)=\mathcal{P}_{r}(v, u)>0, \quad \int \mathcal{P}_{r}(u, v) d \mu(v)=1=\int \mathcal{P}_{r}(u, v) d \mu(u)
$$

Proof The first equality is a consequence of the kernel $\mathcal{P}_{r}$ definition. Putting $f \equiv 1$ in (8.1) and using the first equality, we obtain the other equalities.

Theorem 8.3 For every $f \in L_{\mu}^{p}(1 \leq p \leq \infty)$, we have $\left\|\mathcal{P}_{r}[f]\right\|_{L_{\mu}^{p}} \leq\|f\|_{L_{\mu}^{p}}$ for all $r \in[0,1)$. If, in addition, $1 \leq p<\infty$, then

$$
\lim _{r \rightarrow 1}\left\|\mathcal{P}_{r}[f]-f\right\|_{L_{\mu}^{p}}=0, \quad f \in \mathcal{H}_{\mu}^{p}
$$

Proof First, note that the invariant property (4.3) yields

$$
\mathcal{P}_{r}[f](v)=\int \mathcal{P}_{r}\left(\mathbb{1}, v^{-1} u\right) f(u) d \mu(u)=\int \mathcal{P}_{r}(\mathbb{1}, s) f(v s) d \mu(s), \quad f \in L_{\mu}^{\infty} .
$$

So, if $p=\infty$, then $\left\|\mathcal{P}_{r}[f]\right\|_{L_{\mu}^{\infty}} \leq\|f\|_{L_{\mu}^{\infty}} \int \mathcal{P}_{r}(\mathbb{1}, s) d \mu(s)=\|f\|_{L_{\mu}^{\infty}}$ for all $f \in L_{\mu}^{\infty}$.

Let $1 \leq p<\infty$. Using the Jensen inequality and the Fubini theorem, we get

$$
\left\|\mathcal{P}_{r}[f]\right\|_{L_{\mu}^{p}} \leq \int\left(\int|f(v u)|^{p} d \mu(v)\right)^{1 / p} \mathcal{P}_{r}(\mathbb{1}, u) d \mu(u) \leq\|f\|_{L_{\mu}^{p}}
$$

for all $f \in C_{b}(U(\infty))$. Via the denseness of $C_{b}(U(\infty))$, this inequality holds for all $f \in L_{\mu}^{p}$. 
By Lemma 8.2, $\mathcal{P}_{r}[f](v)-f(v)=\int[f(v u)-f(v)] \mathcal{P}_{r}(\mathbb{1}, u) d \mu(u)$. Replacing in the previous reasoning $\mathcal{P}_{r}[f]$ by $\mathcal{P}_{r}[f]-f$, we similarly get

$$
\left\|\mathcal{P}_{r}[f]-f\right\|_{L_{\mu}^{p}} \leq \int\left(\int|f(v u)-f(v)|^{p} d \mu(v)\right)^{1 / p} \mathcal{P}_{r}(\mathbb{1}, u) d \mu(u)
$$

for all $f \in L_{\mu}^{p}$. Under the continuity of the shift operator in $L_{\mu}^{p}(1 \leq p<\infty)$, for every $r \in$ $[0,1)$, there exists $\delta>0$ such that $\int|f(v u)-f(v)|^{p} d \mu(v) \leq(1-r)^{p}$ for all $u \in U(\infty)$ such that $\operatorname{Re} \phi_{e}(u)<\delta$. On the other hand, if $r \rightarrow 1$, then for every $\delta>0$, uniformly on $u, v \in U(\infty)$ such that $\operatorname{Re} \phi_{e}\left(v^{-1} u\right) \geq \delta$,

$$
\mathcal{P}_{r}(v, u)=\frac{1-r^{2}}{1-2 r \operatorname{Re} \phi_{e}\left(v^{-1} u\right)+r^{2}\left|\phi_{e}\left(v^{-1} u\right)\right|^{2}} \leq \frac{1-r^{2}}{1-r^{2}-2 r \operatorname{Re} \phi_{e}\left(v^{-1} u\right)} \rightarrow 0 .
$$

It immediately follows that

$$
\int_{\operatorname{Re} \phi_{e}(u) \geq \delta} \mathcal{P}_{r}(\mathbb{1}, u) d \mu(u) \rightarrow 0 \quad \text { as } r \rightarrow 1
$$

This proves the existence of the required limit relation (8.2) for all $f \in \mathcal{H}_{\mu}^{p}$.

Theorem 8.4 For all functions $f \in \mathcal{H}_{\mu}^{\infty}$ and $\eta \in L_{\mu}^{1}$,

$$
\lim _{t \rightarrow 1} \int \mathcal{P}_{r}[f] \eta d \mu=\int f \eta d \mu .
$$

Proof Using the Fubini theorem and Theorem 8.3 in the case $p=1$, we obtain

$$
\begin{aligned}
\int \mathcal{P}_{r}[f] \eta d \mu & =\iint \mathcal{P}_{r}(v, u) f(u) d \mu(u) \eta(v) d \mu(v) \\
& =\iint \mathcal{P}_{r}(v, u) \eta(v) d \mu(v) f(u) d \mu(u) \rightarrow \int \eta f d \mu
\end{aligned}
$$

for any function $\eta \in L_{\mu}^{1}$.

Remark 8.1 The limit relation (8.2) holds for any $f \in L_{\mu}^{p}(1 \leq p<\infty)$. As well, (8.3) holds for any $f \in L_{\mu}^{\infty}$. However, in these cases the approximating functions $\mathcal{P}_{r}[f]$ are not analytic but harmonic in a suitable meaning.

\section{Competing interests}

The author declares that he has no competing interests.

\section{Acknowledgements}

I am grateful to the referees for their comments. This work was partially supported by the Center for Innovation and Transfer of Natural Sciences and Engineering Knowledge at the University of Rzeszów.

Received: 3 December 2015 Accepted: 21 January 2016 Published online: 02 February 2016

\section{References}

1. Gross, L: Abstract Wiener spaces. In: Doebner, HD (ed.) Proc. 5th Berkeley Symp. Math. Stat. and Probab., vol. 2, part 1, pp. 31-42 (1965)

2. Neretin, Y: Hua-type integrals over unitary groups and over projective limits of unitary groups. Duke Math. J. 114(2) 239-266 (2002) 
3. Olshanski, G: The problem of harmonic analysis on the infinite-dimensional unitary group. J. Funct. Anal. 205, 464-524 (2003)

4. Cole, B, Gamelin, TW: Representing measures and Hardy spaces for the infinite polydisk algebra. Proc. Lond. Math. Soc. 53, 112-142 (1986)

5. Neeb, KH, Ørted, B: Hardy spaces in an infinite dimensional setting. In: Doebner, HD (ed.) Lie Theory and Its Applications in Physics, vol. 2, pp. 3-27. Word Scientific, Singapore (1998)

6. Pinasco, D, Zalduendo, I: Integral representations of holomorphic functions on Banach spaces. J. Math. Anal. Appl. 308, 159-174 (2005)

7. Carando, D, Dimand, V, Muro, S: Coherent sequences of polynomial ideals on Banach spaces. Math. Nachr. 282(8), 1111-1133 (2009)

8. Floret, K: Natural norms on symmetric tensor products of normed spaces. Note Mat. 17, 153-188 (1997)

9. Hervé, M: Analyticity in Infinite Dimensional Spaces. de Gruyter, Berlin (1989)

10. Berezansky, YM, Kondratiev, YG: Spectral Methods in Infinite-Dimensional Analysis. Springer, Berlin (1995)

11. Tomas, E: On Prohorov's criterion for projective limits. In: Partial Differential Equations and Functional Analysis. Operator Theory: Adv. Appl., vol. 168, pp. 251-261 (2006)

12. Lopushansky, O: Hardy-type space associated with an infinite-dimensional unitary matrix group. Abstr. Appl. Anal. 2013, Article ID 810735 (2013)

13. Voiculescu, DV: Limit laws for random matrices and free products. Invent. Math. 104, 201-220 (1991)

14. Stroock, DW: Probability Theory: An Analytic View. Cambridge University Press, Cambridge (2010)

15. Rudin, W: Function Theory in the Unit Ball of $\mathbb{C}^{n}$. Springer, Berlin (1980)

\section{Submit your manuscript to a SpringerOpen ${ }^{\ominus}$ journal and benefit from:}

- Convenient online submission

Rigorous peer review

- Immediate publication on acceptance

- Open access: articles freely available online

- High visibility within the field

- Retaining the copyright to your article 